\title{
Methylmalonic Acidemia and Megaloblastic Anemia due to Congenital Intrinsic Factor Deficiency
}

\author{
Robles ${ }^{* 1}$ and Sanchez-Valle $\mathrm{A}^{2}$
}

${ }^{1}$ University of South Florida, Morsani College of Medicine, Department of Pediatrics, Tampa, Florida, USA

${ }^{2}$ University of South Florida, Morsani College of Medicine, Department of Pediatrics, Division of Genetics and Metabolics, Tampa, Florida, USA

${ }^{*}$ Corresponding author: Robles J, University of South Florida, Morsani College of Medicine, Department of Pediatrics, 2 Tampa General Circle- Room 5036, Tampa, Florida, USA 33606, E-mail: jcastil1@health.usf.edu

Citation: Robles J, Sanchez-Valle A (2017) Methylmalonic Acidemia and Megaloblastic Anemia due to Congenital Intrinsic Factor Deficiency. J Case Rep Stud 5(2): 202. doi: 10.15744/2348-9820.5.202

Received Date: February 13, 2017 Accepted Date: April 25, 2017 Published Date: April 27, 2017

\begin{abstract}
Causes of vitamin B12 deficiency in children include decreased intake, abnormal absorption, and inborn errors of B12 transport and metabolism. Rare causes of abnormal cobalamin absorption include Imerslund-Grasbeck syndrome (IGS) and intrinsic factor deficiency (IFD). IGS and IFD are caused by defects in the genes CUBN, $A M N$, and GIF. We describe a 2 year old male who presented with severe megaloblastic anemia and methylmalonic acidemia. He was found to have a GIF heterozygous mutation $c .79+1 \mathrm{G}>\mathrm{A}$ associated with congenital gastric intrinsic factor deficiency and a novel variant c.960C $>\mathrm{A}$ in trans position. Few cases with his particular mutation and megaloblastic anemia have been reported. His unique presentation was characterized by an initial suspicion of myelodysplastic syndrome and a substantial increase in methylmalonic acid levels, which raised concerns about an inborn error of metabolism.
\end{abstract}

Keywords: Methylmalonic Acidemia; Megaloblastic Anemia; Intrinsic Factor Deficiency

List of Abbreviations: CUBN: Cubilin; AMN: Amnionless Associated Transmembrane Protein; GIF: Gastric Intrinsic Factor; CBC: Complete Blood Count

\section{Introduction}

Causes of vitamin B12 deficiency in children include decreased intake, abnormal absorption, and inborn errors of B12 transport and metabolism. Rare causes of cobalamin malabsorption include Imerslund-Grasbeck syndrome (IGS) and intrinsic factor deficiency (IFD). IGS, in which cobalamin absorption is interrupted and not corrected by intrinsic factor administration, has been associated with defects in the intrinsic factor vitamin B12 receptor gene CUBN and the amnionless gene AMN [1]. IGS was first described in 1960 with recessive inheritance pattern, failure to thrive, low serum cobalamin, infections, megaloblastic anemia, proteinuria and variable neurological symptoms [2].

IFD is caused by mutations in the gene GIF [3]. Patients with IFD present with pancytopenia and megaloblastic anemia and unlike IGS, cobalamin malabsorption is corrected by intrinsic factor administration [3,4]. In 2004, a specific GIF mutation was first identified as a cause of severe anemia and cobalamin deficiency in an 11 year old girl [3]. We describe a 2 year old male who presented with megaloblastic anemia and methylmalonic acidemia, leading to a diagnosis of congenital gastric intrinsic factor deficiency through genetic testing. Few cases have been described with our patient's specific GIF heterozygous mutation c.79+1G $>$ A and no cases with the novel variant c.960C $>$ A [1,4]. His presentation with markedly elevated methylmalonic acid initial suspicion of myelodysplastic syndrome is unique.

\section{Case Presentation}

A 2 year old Caucasian male presented with daily persistent emesis, pallor, and perioral cyanosis. Past medical history was significant for two prior episodes of pneumonia and wheezing. His medications included inhaled beclomethasone dipropionate. His diet included meats, dairy, fruits and vegetables and the family lived in a home built in 2004; to their knowledge the family had no lead exposure. On physical examination, the patient appeared well developed with weight, height and head circumference in the $10^{\text {th }}-25^{\text {th }}$ percentiles. He was pale and had a $2 / 6$ systolic ejection murmur but the exam was otherwise unremarkable. 
Initial evaluation revealed severe megaloblastic anemia. He was referred and had an extensive work-up by hematology including a bone marrow biopsy, which led to the initial suspicion for myelodysplastic syndrome; cytogenetic testing was negative. Further laboratory work-up revealed markedly elevated methylmalonic acid prompting a referral to metabolic genetics. Homocysteine and methylmalonic acid levels were increased while vitamin B12 levels were decreased (Table 1).

\begin{tabular}{|c|c|}
\hline Laboratory Study & Result \\
\hline Complete Blood Count & WBC 6.4 x10E3/uL (ref. 4.86-13.38) \\
& $\begin{array}{c}\text { Hemoglobin 5.3g/dL (ref. 10.2-12.7) } \\
\text { MCV 105fL (ref.71-85) } \\
\text { RDW 26.8 (12-14.9) }\end{array}$ \\
\hline Lactate dehydrogenase & Platelet count 115x10E3/uL (ref. 150-350) \\
\hline Fanconis DNA & 9574 IU/L (ref.500-920) \\
\hline Bone marrow cytogenetics & No mutation detected \\
\hline Serum Methylmalonic acid level & 46, XY \\
\hline Urine organic acids & Marked elevation of methylmalonate. Methylcitrate \\
& and 3-OH propionate were also present but not in \\
& elevated amounts \\
\hline Serum amino acids & Normal \\
\hline Acylcarnitine profile & C3 of 5.84umol/L (ref 0.14-0.85) and elevated C3/C2 \\
ratio
\end{tabular}

Table 1: Patient's Laboratory Results

Genetic testing of a megaloblastic anemia panel for genes CUBN, AMN, and GIF were sent to Centogene AG in Germany; the patient was found to have a GIF heterozygous mutation c.79+1G>A and a heterozygous variant c.960C >A (p. N320 K). The GIF heterozygous mutation in intron $1(\mathrm{c} .79+1 \mathrm{G}>\mathrm{A})$ was reported as disease-causing as it disrupts the highly conserved donor splice site therefore predicted to highly likely have an aberrant impact on the splicing process. The heterozygous variant c.960C $>$ A (p. $\mathrm{N} 320 \mathrm{~K}$ ) is located in a moderately conserved nucleotide and amino acid position with moderate physiochemical differences between the amino acids lysine and asparagine. Software analysis by SIFT predicted that the variant was benign whereas Polyphen 2 indicated the variant was probably damaging. Since it was unclear if the variant was pathogenic or non-pathogenic, the parents and sibling were tested. The heterozygous mutation was found in the father and the heterozygous variant mutation was found in the mother. Centogene AG predicted his genetic testing was consistent with a diagnosis of intrinsic factor deficiency due to the presence of two changes in the GIF gene. A CUBN, heterozygous variant in exon 19 c. 2594 G>A (p.S865N) was also found but predicted to be a neutral polymorphism. No pathogenic mutation of $A M N$ was found.

The patient was started on weekly $1 \mathrm{mg}$ cobalamin injections. His hemoglobin, methylmalonic acid, homocysteine, and cobalamin levels normalized over two months. He was given a trial off of the injections and his vitamin B12 levels started to decline; cobalamin injections were resumed.

\section{Discussion}

Congenital intrinsic factor deficiency has been reported as a rare cause of megaloblastic anemia [1,3]. The genetic testing of the $C U B N, G I F$, and $A M N$ genes was solely available through a genetic laboratory in Germany. The GIF mutation (c.79+1G>A) has been described as a loss of function mutation, first identified in a French family clinically diagnosed with IGS; genetic testing revealed that the family had IFD [1]. In the French family, the patients were diagnosed at 1.5 and 6 years of age with proteinuria, decreased cobalamin levels, and megaloblastic anemia. The GIF splice site mutation (c.79+1G>A) was also found in a 15 year old boy with megaloblastic anemia with pancytopenia, slight proteinuria and slightly elevated methylmalonate [4].

The patients with this GIF mutation presented with megaloblastic anemia, similarly to our patient. While one of these patients (the 15 year old boy previously mentioned) had slightly elevated methylmalonic acid, the methylmalonic acid level in our patient was 80 times higher than the upper limit of normal leading to a suspicion for isolated methylmalonic acidemia. Isolated methylmalonic acidemia or aciduria may develop due to a deficiency of the methylmalonyl-CoA mutase or methylmalonyl-CoA epimerase caused by mutations in genes MUT, MMAA, MMAB, MCEE, and MMADHC [5]. These mutations result in a range of clinical manifestations from a severe neonatal presentation to an intermediate adult presentation [5]. This was considered in our patient until his laboratory results revealed elevated homocysteine levels and indicated vitamin B12 deficiency as a cause of his megaloblastic anemia, thrombocytopenia, and methylmalonic acidemia. Increased methylmalonic acid and homocysteine levels are expected and highly sensitive indicators of vitamin B12 deficiency [6]. 
The initial working diagnosis for our patient was myelodysplastic syndrome due to his megaloblastic anemia and the appearance of his bone marrow. This presentation of Congenital IFD is unique and has not been previously reported. Preliminary diagnoses of leukemia or myelodysplastic syndrome have been reported in Transcobalamin (TC) deficiency which is a rare autosomal recessive disorder in which infants present with failure to thrive, diarrhea, pallor, and pancytopenia [7]. The defect in TC deficiency interferes with endocytosis of cobalamin by cells and leads to megaloblastic anemia and elevated total plasma homocysteine and methylmalonic acid levels [7]. The similarity in presentation of our patient and those described with TC deficiency support that our patient's laboratory and bone marrow findings leading to an initial diagnosis of myelodysplastic syndrome were indeed the result of his congenital IFD and subsequent cobalamin deficiency.

In children presenting with macrocytic anemia, the differential diagnosis includes myelodysplastic syndrome and bone marrow failure, yet cobalamin and folate deficiency should be considered even in the absence of an obvious explanation. In any child presenting with a possible vitamin B12 deficiency, the vitamin B12, methylmalonic acid and homocysteine levels should be measured [8].

\section{Conclusion}

Vitamin B12 deficiency is an important differential diagnosis in children with megaloblastic anemia and methylmalonic acidemia. Measurement of vitamin B12, methylmalonic acid and homocysteine levels is essential in these children. Genetic testing for congenital intrinsic factor deficiency, specifically for the CUBN, GIF, and $A M N$ genes, should be considered in pediatric patients without a clear etiology of vitamin B12 deficiency or in patients dependent on supplemental cobalamin injections.

\section{Acknowledgement}

We would like to thank our patient and his family for allowing us to present his case to the medical community and Julie Stann and Maria Gomez with the Division of Genetics and Metabolics for contacting the family and obtaining consents. We would also like to thank Dr. Jane Carver with her assistance in reviewing and editing the case report.

\section{References}

1. Tanner SM, Li Z, Perko JD, Oner C, Cetin M, et al. (2005) Hereditary juvenile cobalamin deficiency caused by mutations in the intrinsic factor gene. Proc Natl Acad Sci U S A 102: 4130-3.

2. Grasbeck R, Gordin R, Kantero I, Kuhlback B (1960) Selective vitamin B12 malabsorption and proteinuria in young people. A syndrome. Acta Med Scand 167: 289-96.

3. Yassin F, Rothenberg SP, Rao S, Gordon MM, Alpers DH, et al. (2004) Identification of a 4-base deletion in the gene in inherited intrinsic factor deficiency. Blood 103: 1515-7.

4. Overgaard UM, Tanner SM, Birgens HS (2010) Vitamin B12 deficiency in a 15-year old boy due to mutations in the intrinsic factor gene, GIF. Br J Haematol 150: 369-71.

5. Manoli I, Sloan JL, Venditti CP (2016) Isolated Methylmalonic Acidemia In: GeneReviews(R), ed. RA Pagon, MP Adam, HH Ardinger, et al. Seattle (WA): University of Washington, Seattle.

6. Savage DG, Lindenbaum J, Stabler SP, Allen RH (1994) Sensitivity of serum methylmalonic acid and total homocysteine determinations for diagnosing cobalamin and folate deficiencies. Am J Med 96: 239-46.

7. Trakadis YJ, Alfares A, Bodamer OA, Buyukavci M, Christodoulou J, et al (2014) Update on transcobalamin deficiency: clinical presentation, treatment and outcome. J Inherit Metab Dis 37: 461-73.

8. Rasmussen SA, Fernhoff PM, Scanlon KS (2001) Vitamin B12 deficiency in children and adolescents. J Pediatr 138: 10-7. 